\title{
Hansjörg Herr
}

\section{Das Kaninchen vor der Schlange Europäische Wirtschaftspolitik nach dem Anschlag vom 11. September 2001}

\section{Die ökonomische Entwicklung während der neunziger Jahre}

Die USA erlebten in den neunziger Jahren die längste Aufschwungphase seit dem zweiten Weltkrieg. Die Wachstumsraten des realen Bruttoinlandsproduktes (BIP) lagen höher als in Deutschland (und Europa) und in Japan (vgl. Abbildung 1). Die Wachstumsdynamik in den USA wurde binnenwirtschaftlich durch eine hohe Investitionsnachfrage und vor allem eine hohe Konsumnachfrage angetrieben. Gleichzeitig flossen während der gesamten neunziger Jahre große Summen von Kapital in die USA. Die Kapitalströme erzeugten einen starken US-Dollar und riesige Leistungsbilanzdefizite der USA. Die USA agierten aufgrund der hohen Leistungsbilanzdefizite während der gesamten neunziger Jahre als Lokomotive de Weltkonjunktur: ohne die hohe Importnachfrage der USA wäre die Weltwirtschaft langsamer gewachsen (vgl. Evans/Heine/Herr 2001).

Im Jahr 2000 ging der Aufschwung in den USA zu Ende, seit dieser Zeit befinden sich die USA in einer scharfen Abschwungbewegung mit schnell sinkenden realen Wachstumsraten (vgl. Abbildung 1). Der Terror-Anschlag in New York hat die USA und die Weltwirtschaft somit zu einem äußerst ungünstigen Zeitpunkt getroffen. Denn es ist davon auszugehen, dass der Anschlag den Abwärtstrend beschleunigt. Zwar sind die direkten ökonomischen Kosten des Anschlags in keiner Weise ausreichend, eine Krise zu verstärken, jedoch gingen von dem Anschlag negative Vertrauenseffekte aus, die die Investitions- und die Konsumnachfrage nachhaltig dämpfen können. Die Aktionen der westlichen Welt im Rahmen des „Krieges gegen den Terror“ sowie die Gefahr weiterer Anschläge sind nicht geeignet, das Niveau der Unsicherheit zu vermindern. In einer Situation hoher Unsicherheit über die zukünftige Entwicklung sind abrupte und starke Erwartungsänderungen, die durch Zufälligkeiten ausgelöst werden können, nicht auszuschließen. Sollten sich die Unsicherheiten nicht reduzieren oder würde die Welt von weiteren nega- 
tiven Erwartungsschock getroffen werden, dann wäre eine tiefe, weltweite Rezession nicht zu verhindern. ${ }^{1}$

Abbildung 1: Die Wachstumsraten des realen BIPs in Prozent

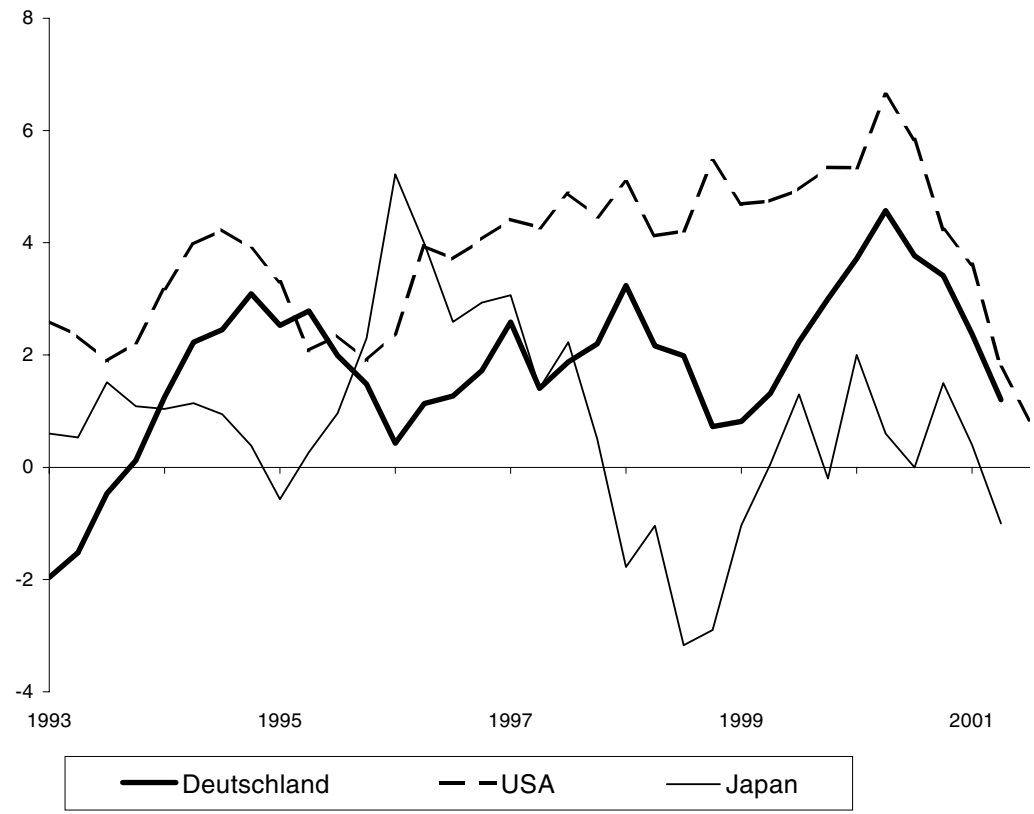

Quelle: OECD, Historical Statistics; IMF, Economic Outlook

Verstärkt durch den Terror-Anschlag wird in den USA im Jahre 2001 ein reales Wachstum von nur 1\% erwartet (vgl. Tabelle 1). In den beiden letzten Quartalen des Jahre 2001 schrumpft die US-Ökonomie und befindet sich somit in einer Rezession. Wieweit im Jahre 2002 in den USA tatsächlich eine Wachstumsrate des BIPs von 1\% erreicht wird, ist zweifelhaft, da die Prognose durch äußerst hohe Unsicherheiten geprägt ist.

1 „Besonders in anormalen Zeiten, wenn die Hypothese einer unbegrenzten Dauer der bestehenden Geschäftslage weniger einleuchtend ist als gewöhnlich, obschon keine besonderen Gründe für die Vorwegnahme einer bestimmten Änderung bestehen, wird der Markt Wellen von Optimismus und Pessimismus ausgesetzt sein, die unvernünftig und doch in einem Sinne gerechtfertigt sind, wenn keine solide Grundlage für eine vernunftgemäße Berechnung besteht." (Keynes 1936, S. 130) 
Tabelle 1: Prognose für das Wachstum des realen BIPs

\begin{tabular}{|c|c|c|c|c|c|c|c|}
\hline & Welt & OECD & EU & Euroraum12 & USA & Japan & $\begin{array}{c}\text { Welthandel } \\
\text { (Waren) }\end{array}$ \\
\hline 1999 & 3,6 & 3,1 & 2,6 & 2,6 & 4,1 & 0,8 & 6,2 \\
\hline 2000 & 4,7 & 4,1 & 3,4 & 3,4 & 4,1 & 1,5 & 12,9 \\
\hline 2001 & 2,0 & 1,0 & 1,7 & 1,6 & 1,0 & $-0,7$ & 0,3 \\
\hline 2002 & 2,2 & 1,2 & 1,8 & 1,6 & 1,0 & $-0,5$ & 2,3 \\
\hline
\end{tabular}

Quelle: Euroframe 2001

Die sowieso schwache konjunkturelle Entwicklung in Deutschland und Europa wurde in den neunziger Jahren im Gegensatz zu den USA wesentlich durch steigende Exporte getragen. Deutschland realisierte im Jahre 2000 noch einen Anstieg der realen Exporte von 13,2\%, im Jahre 2001 ist nur noch ein Wachstum von 4,7\% und im Jahre 2002 von 2,3\% zu erwarten. Nach Prognosen der sechs führenden Wirtschaftsforschungsinstitute Deutschlands sinkt der reale Außenbeitrag Deutschlands von 102,8 Mrd. DM im Jahre 2000 auf 89,4 Mrd. DM ein Jahr später. Von anderen Nachfragekomponenten ist in Deutschland ebenfalls keinerlei Stimulierung zu erwarten, da die Anlageinvestitionen im Jahre 2001 schrumpfen und auch die Konsumnachfrage schwach ist (vgl. DIW 2001, S. 686). Mit der Krise der USÖkonomie stockt auch das Wachstum in Europa. Im Euroraum wird 2001 ein Wachstum von 1,6\% erwartet, in Deutschland von deutlich unter einem Prozent. Der Sachverständigenrat erwartet in seinem Gutachten für das Jahr 2002 eine Wachstumsrate in Deutschland in Höhe von 0,7\%, jedoch ist auch diese Prognose mit hohen Unsicherheiten verbunden (vgl. Tagesspiegel vom 14. November 2001). Faktisch ist die deutsche Wirtschaft Ende 2001 durch Nullwachstum geprägt und steht am Rande einer Rezession. Vom konjunkturellen Abschwung ist Ostdeutschland als unterentwickelte Region stärker betroffen als Westdeutschland. Selbst wenn der Abschwung in Deutschland im Jahre 2002 gestoppt wird und die Ökonomie sich positiv entwickelt, wird für 2002 für Ostdeutschland ein Anstieg der offiziellen Arbeitslosenquote auf 17,1\% (ein Jahr davor 16,7\%) erwartet; in Westdeutschland verharrt die Arbeitslosenquote im besten Fall bei 7,4\%. Ein Abbau der Arbeitslosigkeit ist selbst bei positiven Prognosen auf Jahre verschoben (vgl. DIW 2001).

Japan befindet sich schon während der gesamten neunziger Jahre in einer labilen Verfassung mit teilweise schrumpfender Ökonomie (vgl. Abbildung 1). Japan befindet sich 2001 in einer Rezession; auch im kommenden Jahr wird es Japan nicht gelingen, die Rezession zu überwinden.

Die gegenwärtige weltwirtschaftliche Situation erhält ihre Brisanz dadurch, dass die drei größten Ökonomien der Welt gleichzeitig am Rande einer Rezession stehen bzw. sich in einer Rezession befinden. Dadurch wird die Weltwirtschaft massiv belastet, was sich in der Stagnation des Welthandels im 
Jahre 2001 ausdrückt (vgl. Tabelle 1). Es gibt keinerlei Garantie dafür, dass die positiven Prognosen etwa von Euroframe für das Jahr 2002 zutreffen. Die Lage ist labil. Das Abgleiten in eine sich verfestigende Stagnation oder gar eine tiefe Rezession ist nicht auszuschließen. Die Folgen einer solchen Entwicklung für die sowieso hochverschuldeten Länder der Dritten Welt wären verheerend. Nicht nur Argentinien steht am Rande der Zahlungsunfähigkeit. Die Lage der Weltwirtschaft ist so gefährlich, dass eine aktive wirtschaftspolitische Strategie zur Verhinderung von Stagnation und Rezession insbesondere in den großen Länder der Welt eine Selbstverständlichkeit darstellen sollte. In der Tat reagieren die USA auf die ökonomische Entwicklung aktiv mit expansiver Geld- und Fiskalpolitik (vgl. den Beitrag von Evans in diesem Heft). In der Eurozone ist von einer aktiven Wirtschaftspolitik wenig zu spüren. Hier reagierten und reagieren sowohl Geld- als auch Fiskalpolitik sehr verhalten und hoffen, dass es der Markt oder die USA mit ihrer Wirtschaftspolitik schon richten. Märkte sind jedoch durch Mechanismen geprägt, die Abschwünge endogen verschärfen und beschleunigen können. Die USA sind nicht stark genug, die Weltwirtschaft alleine zu stabilisieren. Davon, dass in der Eurozone versucht wird, einen Beitrag zu Stabilisierung der ökonomischen Lage zu leisten, ist bisher allerdings nichts zu spüren.

\section{Die europäische Geldpolitik}

Die konjunkturelle Entwicklung zeigte in den USA Ende der neunziger Jahre Überhitzungserscheinungen mit ansteigenden Inflationsraten. Dazu kam ein bis dahin nicht für möglich gehaltener Anstieg der Börsenkurse. Die USamerikanische Zentralbank (Fed) reagierte auf diese Entwicklungen ab 1999 mit langsamen kontinuierlichen Zinssatzerhöhungen (vgl. Abbildung 2). Der Versuch einer sogenannten „weichen Landung“ der Konjunktur misslang, denn der Abschwung gestaltet sich hart. In Abbildung 2 wird deutlich, wie flexibel die Fed auf den hereinbrechenden Abschwung reagiert hat. Beginnend Anfang 2001 wurden die Zinssätze im Laufe des Jahres von über 6,5\% auf unter $2 \%$ abgesenkt.

Die Europäische Zentralbank (EZB) reagierte auf die Erhöhung der Zinssätze in den USA im Jahr 2000 mit eigenen Zinssatzerhöhungen. Die Differenz zwischen dem Geldmarktzinssatz in den USA und dem Geldmarktzinssatz im Euroraum blieb bis Ende 2000 in etwa stabil. Danach senkte die Fed jedoch die Zinssätze, ohne dass sich die EZB dieser Entwicklung anschloss. Im Verlaufe des Jahres 2001 drehte sich die Relation bei den Zinssätzen um - der Zinssatz im Euroraum wurde höher als der Zinssatz in den USA. 
Abbildung 2: Entwicklung der Geldmarktzinssätze in den USA und in der Eurozone und der Wechselkurs des US-Dollar zum Euro

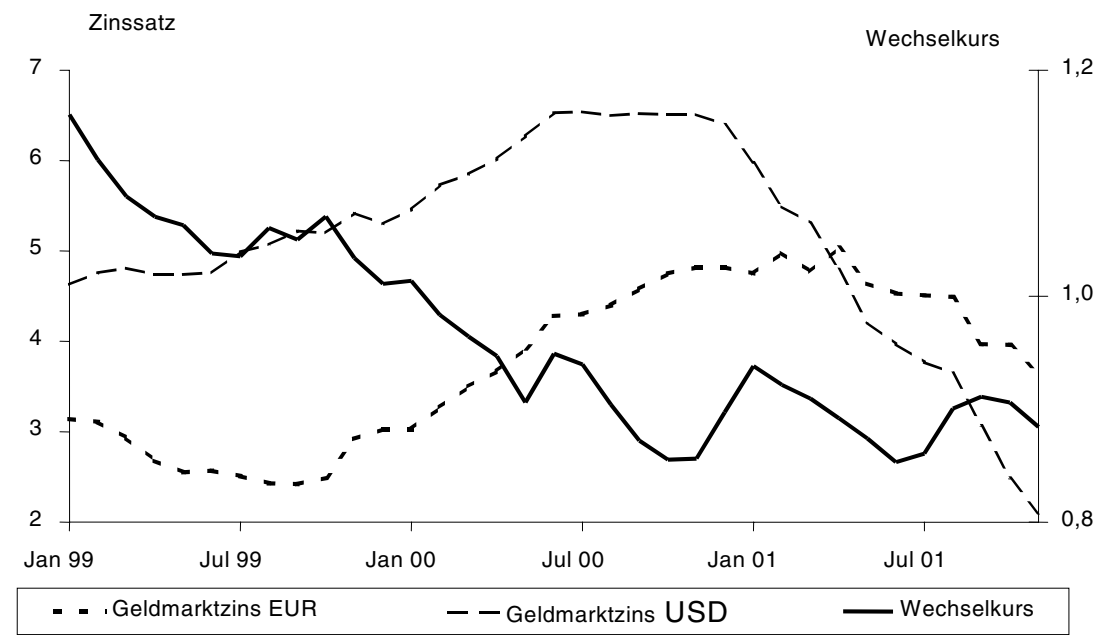

Quelle: EZB, Federal Reserve Bank of St. Louis (USA)

Bis Mitte 2000 zeigte der Euro Schwächezeichen. Seit seiner Einführung im Januar 1999 verlor er gegenüber dem US-Dollar bis zu diesem Zeitpunkt rund 25\% seines Wertes (vgl. Abbildung 2). Die Geldpolitik der EZB in den Jahren 1999 und 2000 lässt sich als eine Strategie interpretieren, welche die Zinsdifferenzen zu den USA angesichts eines schwachen Euro nicht vergrößern wollte. Denn es wäre in dieser Phase nicht auszuschließen gewesen, dass angesichts der Abwertung des Euro eine Politik sinkender Zinssätze seitens der EZB zu kumulativen Kapitalabflüssen und verstärkten Abwertungstendenzen des Euro geführt hätte. Eine starke Abwertung des Euro hätte jedoch von der EZB nicht hingenommen werden können, da dies zu einem Preisniveauschub über steigende Importpreise, zu einem Wohlfahrtsverlust in der Form sinkender Realeinkommen sowie einer weiteren Krise des Vertrauens in den Euro geführt hätte.

Unverständlich bleibt jedoch, dass sich die EZB nicht sofort den Zinssenkungen in den USA Anfang 2001 angeschlossen hat. Denn ab Mitte 2000 ist der Euro gegenüber dem US-Dollar abgesehen von kleinen Schwankungen stabil geblieben. Zumindest hätte die Möglichkeit bestanden, auszutesten, ob eine Zinssenkung im Euroraum im Gefolge der Zinssenkungen in den USA möglich gewesen wäre, ohne dass es zu massiven Kapitalabwanderungen gekommen wäre. Notwendig wäre eine Zinssenkung gewesen, denn die Konjunktur stürzte in Europa in der gleichen Weise und zum gleichen Zeitpunkt 
ab wie in den USA (vgl. Abbildung 1). Durch das zögerliche Verhalten der EZB wurden frühe Zinssenkungen verpasst und der Abschwung nahm auch in Europa seinen Lauf.

Die EZB begründete ihre Politik hoher Zinssätze mit Inflationsgefahren. Sie definierte Preisniveaustabilität in ihrem ersten Monatsbericht im Januar 1999 als eine mittelfristig zu erreichende Inflationsrate zwischen $0 \%$ und 2\% (EZB 1999). Eine mittelfristig zu verteidigende Inflationsgrenze von $2 \%$ ist im internationalen Vergleich äußerst restriktiv. Beispielsweise hat sich die Fed keine quantitativen Vorgaben bei der zu erreichenden Inflationsrate gesetzt und ist damit in ihren Handlungen flexibler als die EZB. Eine Zielinflationsrate von nicht höher als 2\% ist für die ökonomische Entwicklung dysfunktional, da kräftige durch hohe Investitionen getragene Aufschwünge in aller Regel mit Inflationsraten verbunden sind, die temporär über 2\% ansteigen. Die Durchsetzung einer sehr niedrigen Inflationsrate während konjunktureller Aufschwünge impliziert eine Geldpolitik, welche Aufschwünge verhindert, die Ökonomie in einer Halbstagnation verharren lässt und langfristig das Wachstum bremst. Damit soll nicht gesagt werden, Zentralbanken hätten nicht für Preisniveaustabilität zu sorgen. Das Preisniveauziel sollte jedoch so verfolgt werden, dass konjunkturelle Aufschwünge nicht zu schnell durch steigende Zinssätze abgewürgt werden. Zentralbanken sollten bei ihrer Geldpolitik immer berücksichtigen, ob die Gefahr einer sich beschleunigenden Inflation besteht oder ob sich die Inflationsrate auch ohne restriktive Geldpolitik wieder zurückbildet. Mechanisch angewandte Regeln bei der Formulierung der Geldpolitik - etwa: die Inflationsrate darf nicht über 2\% ansteigen - sind dysfunktional und wachstumsfeindlich.

Betrachtet man die Entwicklung der Inflationsrate seit der Einführung des Euro, dann ergibt sich folgendes Bild: Die Inflationsrate lag zunächst unter $2 \%$, stieg jedoch in der zweiten Hälfte des Jahres 2000 über die Grenze von 2\%, um sich Ende 2001 wieder der Grenze von 2\% zu nähern. Für das Jahr 2002 wird eine Inflationsrate von 1,6\% prognostiziert (vgl. Euroframe 2001). Hinter dem temporären Anstieg der Inflationsrate verbergen sich jedoch keine anhaltenden Inflationsgefahren, die eine Zentralbank zu restriktiver Geldpolitik bewegen sollten. Es waren Sonderfaktoren wie steigende Ölpreise, steigende Steuern und steigende Fleischpreise aufgrund der BSE-Krise, die einen Preisniveauschub im Jahre 2000 auslösten.

Inflationsprozesse sind immer durch eine Lohn-Preis-Spirale gekennzeichnet, die ein gegenseitiges Aufschaukeln von Preisen und Lohnstückkosten beinhaltet. ${ }^{2}$ Ein solcher Prozess war im Euroraum nicht zu erkennen, da die Lohnstückkosten keine inflationären Gefahren signalisierten. Vielmehr verhal-

2 Vgl. zur Analyse von Inflationsprozessen und auch zu anderen theoretischen Punkten, die hier nicht im einzelnen ausgeführt werden können, Heine/Herr (2000). 
ten sich die Gewerkschaften in allen großen europäischen Ländern kooperativ und verfolgen eine produktivitätskostenorientierte und damit preisniveauneutrale Lohnpolitik oder schließen gar Tarifverträge ab, die Lohnerhöhungen unter dem Produktivitätsfortschritt festschreiben. Auch war zu keinem Zeitpunkt seit dem Bestehen der EZB eine überschäumende Konjunktur zu erkennen, die einen nachfragebedingten Anstieg des Preisniveaus hätte erklären können. Angesichts der hereinbrechenden Konjunkturkrise sind nächstes Jahr keine expansiven Lohnerhöhungen im Euroraum zu erwarten. Kurz: Es gab keine Inflationsgefahren, die insbesondere seit der Stabilisierung des Wechselkurses des Euro einer Zinssenkung der EZB entgegen gestanden hätten.

Ohne Zweifel hat die Geldpolitik der EZB ab Ende 2000 restriktiv und wachstumsmindernd gewirkt. Sie war im Vergleich zur Geldpolitik in den USA weniger flexibel und zu zögerlich. Jedoch muss bedacht werden, dass auch eine schnellere geldpolitische Reaktion der EZB den Abschwung nicht hätte verhindern können. Die Macht von Zentralbanken zur Stimulierung von Investitionen und Wachstum während eines ökonomischen Abschwungs ist begrenzt. Eine Zentralbank kann durch Zinssenkungen die Belastung der Schuldner mindern und damit Kaufkraft von Konsumenten freisetzen und den Unternehmenssektor stabilisieren. Auch kann eine Zentralbank durch Zinssenkungen den Kursverfall von Aktien zu bremsen versuchen. Expansive Geldpolitik kann jedoch bei einem zerrütteten Vertrauen der Investoren die Nachfrage nach Produktivvermögen nicht erhöhen. Gerade nach dem Terroranschlag am 11. September 2001 in New York hat sich das Niveau der Unsicherheit erhöht. Die Unternehmen und auch Konsumenten reagieren offensichtlich äußerst vorsichtig und lassen sich derzeit auch durch niedrige Zinsen nicht zu Investitionen und zum Kauf langfristiger Konsumgüter bewegen. Auch weitere Zinssenkungen, so wünschenswert sie sind, werden wohl nicht zu einer schnellen Überwindung der gegenwärtigen Krise führen.

\section{Die europäische Fiskalpolitik}

Scharfe konjunkturelle Abschwünge oder gar Rezessionen rufen die Fiskalpolitik auf den Plan. Selbst wenn langfristig ein ausgeglichenes Budget des Staats angestrebt wird, wird nahezu jeder Ökonom eine antizyklische Fiskalpolitik befürworten. In Abschwüngen muss dann das Budgetdefizit ansteigen (bzw. der Budgetüberschuss abnehmen) und in Aufschwüngen abnehmen (bzw. ein Überschuß zunehmen).

Die USA haben auf die krisenhafte Entwicklung in nahezu lehrbuchmäßiger Form reagiert. Sie haben nicht nur die Zinssätze gesenkt, sondern auch eine Reihe von fiskalischen Maßnahmen in die Wege geleitete, welche die (auch wenn sie vor allem den besser verdienenden Schichten zu Gute kommen, vgl. Evans und Mayer/Greven in diesem Heft) Nachfrage und Produktion stabili- 
sieren (vgl. DIW 2001). Die USA verfolgen somit eine im Grunde keynesianische Strategie koordinierter Geld- und Fiskalpolitik - selbst wenn sie diese Politik nicht so nennen. Eine flexible Fiskalpolitik hat in den USA ein lange Tradition. So nahmen die USA während der konjunkturellen Schwäche Anfang der neunziger Jahre, die nicht in eine Rezession mündete, Budgetdefizite als Anteil am BIP in Höhe von 6,0\% im Jahre 1992, 5,1\% im Jahre 1993, 3,9\% im Jahre 1994 oder 3,3\% im Jahre 1995 hin. Ende der neunziger Jahre wichen die Defizite Budgetüberschüssen (vgl. IMF 2000, S.217). Es gibt keinen Zweifel, dass die USA auch in der gegenwärtigen Krise gegebenenfalls hohe Budgetdefizite hinnehmen, um die Ökonomie zu stabilisieren.

Europa stellt sich gerade auf fiskalischem Gebiet als ein Hort von Inflexibilität und versteinerten Regeln dar. Die USA wären Anfang der neunziger Jahre mit ihrer Fiskalpolitik massiv am Vertrag von Maastricht und am sogenannten Stabilitäts- und Wachstumspakt gescheitert. Der Vertrag von Maastricht, welcher der Europäischen Währungsunion (EWU) zu Grunde liegt, sieht eine Höchstgrenze von Budgetdefiziten für Teilnehmer an der EWU in Höhe von 3\% am BIP vor. Mitte der neunziger Jahre wurde insbesondere auch von deutscher Seite ins Spiel gebracht, dass im Maastricht-Vertrag keine ausreichenden Sanktionen bestehen würden, wenn ein Land erst einmal Mitglied der EWU sei, sich dann jedoch nicht an des 3\%-Kriterium halte. Diesen Mangel sollte der Stabilitäts- und Wachstumspakt, der Ende 1996 vom Europäischen Rat beschlossen wurde, beseitigen. Er beinhaltet im Vergleich zum Maastricht-Vertrag folgende Verschärfungen:

Erstens ist eine Überschreitung der 3\%-Grenze nur erlaubt, wenn ein außergewöhnliches, exogen verursachtes Ereignis oder eine schwere Wirtschaftskrise auftritt. Von letzterer wird in der Regel nur dann ausgegangen, wenn das BIP auf Jahresbasis schneller als $2 \%$ schrumpft. $^{3}$

Zweitens sollen Sanktionen innerhalb von 10 Monaten verhängt werden, wenn ein übermäßiges Defizit festgestellt werden konnte. ${ }^{4}$ Allerdings wird letztlich vom Rat der Finanzminister politisch entschieden, ob ein Ausnahmefall vorliegt, der ein Überschreiten des fiskalischen Referenzwertes ohne Sanktionen erlaubt.

Im Kern erzwingt der Stabilitäts- und Wachstumspakt, dass in der Regel auch in konjunkturellen Abschwüngen die 3\%-Marke nicht überschritten wird und damit der Budgetsaldo überzyklisch deutlich unter der Grenze von drei Pro-

3 Schrumpft das BIP auf Jahresbasis stärker als 0,75\%, dann kann das betroffene Land beantragen, von der 3\%-Regel befreit zu werden.

4 Das Sünderland muss 0,2\% des BIP plus 10\% des den Referenzwert überschreitenden Budgetdefizits als Einlage hinterlegen. Die jährliche Sanktion darf jedoch 0,5\% des BIP nicht überschreiten. Die Einlage muss jährlich wiederholt werden, wenn ein Land über einen längeren Zeitraum den Referenzwert verfehlt. Erreicht ein Land den Referenzwert auch nach 2 Jahren nicht, erhält es die Einlage nicht zurück, da diese in eine Strafe umgewandelt wird. 
zent verbleibt. Die Ausgabenstruktur spielt keine Rolle, da die drei Prozent auch nicht überschritten werden dürfen, wenn öffentliche Investitionen finanziert werden (vgl. Priewe 1997).

Das 3\%-Kriterium ist ökonomisch unsinnig. Denn ein permanentes Budgetdefizit von über 3\% am BIP ist unproblematisch, wenn der Anteil der Staatsschuld am BIP nicht steigt. Länder mit hohem Wachstum können auch langfristig Budgetdefizite von 3\% und höher ohne langfristige Destabilisierungstendenzen realisieren. Bei Ländern mit geringen Wachstumsraten und hohen Budgetdefiziten kann auch ein anhaltendes Budgetdefizit von 2\% am BIP zu hoch sein, da die Staatsverschuldung gemessen als Anteil am BIP beständig ansteigt. Ein anhaltender Anstieg der öffentlichen Schuldenquote (öffentlicher Schuldenbestand am BIP) ist negativ, da damit der Spielraum des Staates eingeengt wird. Auch sind mit steigender Staatsschuld negative Verteilungseffekte verbunden, da die Steuern von der breiten Masse bezahlt werden, während die Zinseinkünfte vor allem an höhere Einkommensbezieher fließen. Jedoch gibt es keine feste „kritische“ Verschuldungsgrenze. So haben eine Reihe von Ländern in Europa weitaus höhere Schuldenquoten als andere Länder, ohne dass sich dadurch eine Destabilisierung ergeben würde. Im Eurowährungsraum lag die durchschnittliche Schuldenquote Ende 2000 knapp unter 70\%. In Italien und Belgien betrug sie über $110 \%$, während sie beispielsweise in Deutschland, Frankreich, Österreich oder Spanien in der Nähe von 60\% lag (vgl. DIW 2001: 29). Die Verschuldungsquoten im Euroraum legen auf keinen Fall nahe, dass man für mehrere Jahre zur Konjunkturstabilisierung nicht expansive fiskalische Impulse setzen könnte.

Vor dem Hintergrund des Stabilitäts- und Wachstumspakts sind die politischen Spielräume für Fiskalpolitik im Euroraum bereits recht eingeschränkt. Tabelle 2 verdeutlicht, dass insbesondere die großen Länder in der EWU schon im Jahre 2001 gefährlich nahe an die 3\%-Grenze herankommen dürften. So haben die drei größten Länder in der EWU im Jahre 2001 Budgetdefizite (Italien 2,7\%, Deutschland 2,5\% und Frankreich 2,0\%), die der Fiskalpolitik keinen großen Spielraum mehr lassen. Es ist zu befürchten, dass in diesen Ländern noch nicht einmal die „automatischen Stabilisatoren“ zum Wirken kommen. Unter automatischen Stabilisatoren wird verstanden, dass sinkende Steuereinnahmen und steigende Ausgaben aufgrund der konjunkturellen Entwicklung von den öffentlichen Haushalten hingenommen werden, was die Konjunktur stabilisiert. Wird sich die konjunkturelle Entwicklung weiter verschlechtern, dann werden die großen Länder in der EWU schnell an die 3\%-Grenze stoßen. Versuchen sie dann, die Budgetdefizite nicht größer werden zu lassen, werden sie Ausgabensenkungen und/oder Steuererhöhungen beschließen müssen. In diesem Fall würde letztlich eine pro-zyklische Fiskalpolitik betrieben, welche zur Verschärfung des konjunkturellen Abschwungs beiträgt. 
Tabelle 2: Finanzierungssaldo der öffentlichen Haushalte in Prozent am BIP (Abgrenzung gemäß dem Vertrag von Maastricht; ohne Sondereinnahmen aus der Vergabe von Mobilfunklizenzen)

\begin{tabular}{|c|c|c|c|c|}
\hline & 1998 & 1999 & 2000 & 2001 \\
\hline Deutschland & $-2,2$ & $-1,6$ & $-1,3$ & $-2,5$ \\
\hline Frankreich & $-2,7$ & $-1,6$ & $-1,3$ & $-2,0$ \\
\hline Italien & $-2,8$ & $-1,8$ & $-1,5$ & $-2,7$ \\
\hline Spanien & $-2,6$ & $-1,1$ & $-0,4$ & $-0,2$ \\
\hline Niederlande & $-0,8$ & 0,4 & 1,5 & 0,2 \\
\hline Belgien & $-0,8$ & $-0,6$ & 0,1 & $-0,1$ \\
\hline Österreich & $-2,4$ & $-2,2$ & $-1,5$ & $-1,3$ \\
\hline Finnland & 1,3 & 1,9 & 6,9 & 3,2 \\
\hline Griechenland & $-2,4$ & $-1,8$ & $-1,1$ & $-1,0$ \\
\hline Portugal & $-2,4$ & $-2,1$ & $-1,8$ & $-2,0$ \\
\hline Irland & 2,3 & 2,3 & 4,5 & 3,0 \\
\hline Luxemburg & 3,5 & 3,7 & 6,1 & 3,8 \\
\hline Euroraum & $-2,2$ & $-1,3$ & $-0,8$ & $-1,6$ \\
\hline gewichtet mit BIP von 2000 & & & & \\
\hline
\end{tabular}

Quelle: DIW (2001: 29)

Theoretisch wäre es möglich, selbst im Rahmen des Stabilitäts- und Wachstumspakts - die entsprechenden Klauseln existieren - durch eine temporäre Aufgabe des 3\%-Kriteriums den Weg für eine funktionale Fiskalpolitik in der EWU zu ebnen. Jedoch haben viele europäischen Regierungen - nicht zuletzt auch die deutsche Regierung - die Reduzierung der Budgetdefizite auf Null auf ihre Fahnen geschrieben und sich selbst durch öffentliche Versprechungen gebunden. Es ist deshalb zu befürchten, dass der Stabilitäts- und Wachstumspakt auch in einer konjunkturellen Krise hochgehalten wird und man allenfalls zähneknirschend höhere Budgetdefizit hinnimmt. Mit einer aktiven und vorausschauenden Fiskalpolitik hat dies dann allerdings nichts mehr zu tun. Die Länder der EWU sind fiskalpolitisch gelähmt.

Notwendig wäre nicht nur die vollständige Akzeptanz der automatischen (fiskalischen) Stabilisatoren, sondern darüber hinaus eine aktive expansive Fiskalpolitik, am günstigsten wären Ausgabenprogramme. Zwar kann auch über den Weg von Steuersenkungen die Nachfrage stabilisiert werden, jedoch wirken Ausgabenprogramme stärker und direkter als Steuersenkungen auf die volkswirtschaftliche Nachfrage. In der gegenwärtigen konjunkturellen Situation wären Ausgabenprogramme im europäischen Maßstab im Bereich der Infrastruktur, der Sanierung der Städte, im Bereich der ökologischen Sanierung oder in ähnlichen Bereichen angesagt.

Die Bedingungen für Fiskalpolitik verbessern sich mit der Größe des Wirtschaftsraumes, da fiskalische Impulse in großen Wirtschaftsräumen in geringerem Maße über steigende Importe ins Ausland verpuffen. So würde eine expansive Fiskalpolitik etwa der Schweiz aufgrund der hohen Importquote 
der Schweiz stark nach außen wirken und nur begrenzt auf die Binnenwirtschaft. Durch die EWU haben sich die Bedingungen für Fiskalpolitik in Europa erheblich verbessert, denn die EWU stellt einen ähnlich großen Wirtschaftsraum dar wie die USA und hat eine ähnlich geringe handelsmäßige Außenverflechtung.

Die europäische Fiskalpolitik leidet an einem strukturellen Problem, welches die verbesserten Möglichkeiten für eine EWU-weite Fiskalpolitik nicht zum Zuge kommen lassen. Fiskalpolitik in der EWU wird nicht koordiniert. In Tabelle 2 kommt deutlich zum Ausdruck, dass eine Reihe kleinerer Länder sich fiskalpolitisch als Trittbrettfahrer verhalten. Denn es sind besonders die kleineren Länder wie die Niederlande, Finnland oder Irland, die geringe Budgetdefizite oder gar Budgetüberschüsse haben. Diese Länder leben von der Nachfragestimulierung in anderen Ländern. Aufgrund ihrer offenen Ökonomien haben sie verständlicherweise kein Interesse, Fiskalpolitik zu betreiben. Die großen Ökonomien in der EWU könnten aber eine fiskalpolitische Kooperation betreiben. Selbst in der gegenwärtigen konjunkturellen Situation in der EWU und der Weltwirtschaft wird über fiskalpolitisch abgestimmte Maßnahmen in Europa faktisch nicht diskutiert. Frankreich hat ein kleines nationales Konjunkturprogramm beschlossen, Deutschland zieht dabei jedoch in keiner Weise mit.

Antizyklische Fiskalpolitik bedeutet, dass die öffentlichen Haushalte dann ihre Ausgaben erhöhen, wenn Unternehmen und private Haushalte ihre Ausgaben senken und umgekehrt. Fiskalpolitisches Handeln muss somit gerade nicht der Logik des privaten Ausgabeverhaltens folgen. Kleine Gebietskörperschaften können eine solche Funktion nicht übernehmen. So ist seit langem bekannt, dass Gemeinden in Deutschland stark prozyklisch agieren. Sind die Steuereinnahmen hoch, dann geben sie viel aus; sind die Steuereinnahmen niedrig, dann werden die Ausgaben gekürzt. Fiskalpolitik muss jedoch makroökonomisch orientiert auf gesamtwirtschaftlicher Ebene angesiedelt sein. Für die EWU bedeutet, dies dass eine Institution geschaffen werden muss, um Fiskalpolitik für die EWU als Ganze zu betreiben.

\section{Die Europäische Währungsunion vor ihrer bislang größten Herausforderung}

Es besteht die Gefahr, dass die Länder der EWU wirtschaftspolitisch versagen. Zwar wird eine europäische Geldpolitik betrieben, jedoch steht der EZB keine Institution auf „gleicher Augenhöhe“ gegenüber, da es weder ein machtvolles Europäisches Parlament, noch eine machtvolle Regierung gibt. Die EZB ist weitaus abgehobener und mächtiger als es die Deutsche Bundesbank je war. Dies erhöht die Gefahr, dass die Geldpolitik in der EWU - wie seit Ende 2000 - dysfunktional betrieben wird. Aber selbst wenn sich die 
EZB auf ein kooperatives wirtschaftspolitisches Regime einlassen wollte, würde sie kaum potente Partner finden, da eine europäische Regierung, starke europäische Gewerkschaften und starke europäische Arbeitgeberverbände nicht existieren.

Zur Gestaltung einer funktionalen Fiskalpolitik in der EWU bedarf es fiskalpolitischer Koordination. Letztlich bedarf es eines europäischen Finanz- und Wirtschaftsministeriums mit eigenen Einnahmen und Ausgaben und der Möglichkeit der eigenen Kreditaufnahme. Es spricht vieles dafür, dass die Länder der EWU in den kommenden Jahren wirtschaftspolitisch versagen und weder für Europa noch für die Weltwirtschaft stabilisierend wirken. Es bleibt zu hoffen, dass aus einer solchen Entwicklung Lehren gezogen werden und aus der bisherigen EWU ein Wirtschaftsraum entsteht, der wirtschaftspolitisch in allen Dimensionen handlungsfähig wird. Anderenfalls wird nicht nur der längerfristige Erfolg des Euro fraglich sein, auch die Arbeitslosigkeit wird sich dann im Euroraum auf hohem Niveau verfestigen, mit all den bekannten negativen Konsequenzen für die abhängig Beschäftigten.

\section{Literatur}

DIW (Deutsches Institut für Wirtschaftsforschung) (2001): Die Lage der Weltwirtschaft und der deutschen Wirtschaft im Herbst 2001, Gemeinschaftsgutachten der sechs führenden Forschungsinstitute Deutschlands, Wochenbericht des DIW, No. 43

Euroframe (Arbeitsgemeinschaft von acht unabhängigen europäischen Wirtschaftsforschungs instituten) (2001): http://www.euro-frame.org

Evans, T./Heine, M./Herr, H. (2001): Weiche Kurse - Harter Fall? Die außenwirtschaftlichen Perspektiven der US-Ökonomie, in: A. Heise (Hrsg.), USA - Modellfall der New Economy?, Marburg, S. $23-40$

EZB (Europäische Zentralbank) (1999): Monatsbericht, Januar

Heine, M./Herr, H. (1999): Verdrängte Risiken der Euro-Einführung, in: WSI-Mitteilungen, Heft 8

Heine, M./Herr, H. (2000): Volkswirtschaftslehre. Paradigmenorientierte Einführung in die Mikro- und Makroökonomie, 2. Auflage, München

IMF (International Monetary Fonds) (2000), Economic Outlook, October

Keynes, J.M. (1936): Allgemeine Theorie der Beschäftigung, des Zinses und des Geldes, Berlin

Priewe, J. (1997): Verschuldungsregeln in der Europäischen Währungsunion, in: WSIMitteilungen, Heft 6 